\title{
Mobile Gesture Interfaces Based on Touch
}

\author{
M. Isomursu*
}

\section{VTT, Technical Research Centre of Finland, Finland}

\begin{abstract}
This paper summarizes the results of a set of field trials where near-field communication technology was used to construct mobile gestural interfaces for (1) mobile Internet content access, and (2) digital service access. All interface solutions are based on touch gestures where real-world objects are touched with a mobile phone. The results are based on trials and concept evaluations involving more than 300 users. The trials have been arranged in real-world settings, where recruited trial users have adopted the services and interfaces in their everyday lives. A wide variety of data collection methods have been used, ranging from automated usage logs to participatory observations and usability testing. As a result, a set of findings related to adoption and design of mobile touch-based interfaces in everyday interactions are constructed.
\end{abstract}

\section{INTRODUCTION}

Radio frequency identification (RFID) technology has been available and used in different contexts for decades. It has been found to be a cheap, simple and relatively robust technology for applications ranging from m-commerce (mobile commerce) in Japan [1] to cattle management in Australia [2]. In recent years, there has been interest in and efforts to integrate RFID readers into standard mobile phones. In Japan, Sony's passive near-field communication (NFC) FeliCa mobile phones have gained popularity, especially in mobile payment [1]. In Europe, the first mobile phone models integrating NFC-readers and related standardization have been available for some years now, although their consumer penetration currently falls far short of Japan. However, both the number of mobile phones embedding NFC functionality and their related markets are expected to experience vast global growth within the next five years [3].

An integrated NFC reader in a mobile phone enables gestural interaction with a target by means of touching. As near-field communication technologies require the target to be in close proximity to the reader, the user interaction requires an active gesture that brings the reader close to its target. The target touched by the user can be a RFID tag, another NFC-enabled device or a smart card. In most cases, the target is embedded in some everyday object, e.g. the tag is attached to a poster or some other printed visual material, or the reading device is integrated into a ticket validation machine or doorframe. Attaching RFID tags to existing objects has been called "retro-fitting" [4], i.e. the process of incorporating new digital functionality into the existing qualities of the object. Embedding RFID tags into everyday objects and the user's environment can be used to create tangible user interfaces (TUI) that allow the users to "grasp and manipulate" digital bits through objects placed in the physical world [5]. Physical objects and environment can be

*Address correspondence to this author at the VTT, Technical Research Centre of Finland, Finland; Tel: +358 408433 871; Fax: +358 20722 2320;

E-mails: minna.isomursu@vtt.fi, minna.isomursu@gmail.com coupled with digital content, services and interactions. Contrary to most tangible user interface technologies [6], NFC-based user interfaces are very easy and cheap to construct. RFID readers and tags are relatively cheap. No special equipment is required to construct an NFC-based user interface similar to the ones described in this paper, as tags can be written to with a normal NFC-enabled mobile phone. The simplest NFC-based user interfaces do not require programming or special technical skills.

A mobile phone is a promising tool for use in touching, as it features audio, visual or tactile feedback, and enables touch gestures to be combined with keypad or voice input. Touch-based user interfaces can also make mobile phones accessible to user groups - the elderly, for example - who cannot use small phone keypads or read their small screens [7].

In this paper, user interfaces and the related functionalities have been divided into two broad categories describing the different kinds of user interaction possibilities enabled through mobile touch interfaces. These categories have emerged during the course of the research presented in this paper. However, it is likely that they do not describe all the possibilities available to mobile interaction designers through the innovative use of touch. For example, Pering, Ballagas and Want [11] have used mobile touch-based gestures and tagged physical objects for configuring devices and interfaces in interactive spaces.

\section{Physical Browsing}

The first category deals with user interfaces that allow the user to access mobile Internet content through the mobile phone by touching a RFID tag embedded into his or her everyday environment. This can be called "physical browsing" [8]. The user touches a tag with a mobile phone, and the mobile phone automatically accesses the mobile Internet content specified by the tag, and presents it to the user through the applications (e.g. a browser) and output modalities available in the phone (e.g. display and audio). Early examples of this kind of interaction were proposed and demonstrated by HP's Cooltown concept [9]. 


\section{Service Interfaces}

The second category comprises user interfaces that are used for embedding mobile service functionalities into the user's everyday environment. The user touches a tag or another NFC device with a phone, triggering the phone to send a service message or parameter to be processed by the service backend system. In this category of interfaces, NFC is used for creating digital service access points. Simple examples of service interfaces include RFID-based public transportation ticketing [10] and mobile payment [1] solutions, where a touch of the reader transfers the action into the background system that then validates and processes the transaction.

\section{MATERIALS AND METHODOLOGY}

The work described in this paper was done in a research project that explored the use of NFC in different application and service contexts. The project was a European cooperation project with 24 partners from both industry and research. The goal of the project was to trial NFC-based applications and services in various domains, including home, health and public urban environments. All the research material used in this paper was collected in the trials and evaluations arranged within this specific project.

As the trials aimed to analyze the use of new application and service concepts, all material collected during the project was collected in real-world settings. This means, for example, that the trial users were volunteers who committed themselves to using the applications and services as a part of their everyday life during the trial period. All trial users received material describing the trial and the research project prior to their participation, and most received hands-on training and were provided with the opportunity to ask questions and give verbal feedback before the actual trial. All trial users gave their written consent. The trial participants were adults, but some of the concept evaluations involved minors. In these cases, the minors were always accompanied by their parents.

None of the participants were paid for their involvement. However, the trials were planned so that the trial users would not incur any extra costs. That is to say, the users did not pay for the data transfer needed to use the services. In addition, they did not pay anything for the services they used, even though they would be chargeable in normal circumstances.

All data collected was made anonymous for the analysis. For this reason, it is impossible to determine whether some of the participants were involved in more than one of the trials. However, the user recruitment processes for each trial were completely separate. If the same persons were accidentally selected to participate in more than one trial, they served as representatives of their specific target groups in those specific trials. We are not aware of instances where one person would have taken part in more than one trial.

The research methodology used to collect the data presented in this paper is based on:

1. constructive research that explored the use of NFC in specific application domains through constructing new device, application and service concepts that contribute towards a better understanding of the possibilities and limitations of mobile touch-based interaction, and

2. field trials, where the novel NFC-based constructs were used in real-world settings in order to collect feedback on the user experience, value creation and ecological impact.

The analysis and results presented in this paper are based on four separate trials that were all arranged during winter 2007. A summary of the trials and the number of users in each trial is presented in Table $\mathbf{1}$.

Table 1. Durations and Number of Users in Separate Trials

\begin{tabular}{|c|c|c|c|}
\hline & $\begin{array}{c}\text { Period } \\
\text { (Duration) }\end{array}$ & $\begin{array}{c}\text { Number of } \\
\text { Users }\end{array}$ & $\begin{array}{c}\text { Usage Time } \\
\text { Per User }\end{array}$ \\
\hline \hline Parking trial & $\begin{array}{c}3 \text { Sep }-1 \text { Nov } 2007 \\
(8 \text { weeks })\end{array}$ & 51 & Whole trial period \\
\hline Theatre trial & $\begin{array}{c}8 \text { Nov }-19 \text { Dec } 2007 \\
(6 \text { weeks })\end{array}$ & 141 & One evening \\
\hline Pub trial & $\begin{array}{c}12 \text { Nov }-31 \text { Dec } 2007 \\
(7 \text { weeks })\end{array}$ & 19 & Whole trial period \\
\hline Restaurant trial & $\begin{array}{c}19 \text { Nov }-31 \text { Dec } 2007 \\
(6 \text { weeks })\end{array}$ & 27 & Whole trial period \\
\hline
\end{tabular}

The trials are described in the following sections.

\section{Parking Trial}

In the parking payment trial, the trial users were able to pay for parking with the help of NFC. The trial users (51 users) attached an NFC tag to the windshield of their car. When they arrived at the spot where they wanted to park their car, they touched the NFC tag and the tag placed on the nearest parking meter to mark the location of the car (see Fig. 1). Additionally, parking tags were attached to roadside lamp posts. When they left with the car, they once again touched the tag on their car to end their use of pay parking. Each touch gesture sent a parameter to the parking payment background system indicating the start or end of the client's parking session. As an additional feature, the user could use the location identification tags to store the location identifier in the memory of their phone. This feature was targeted particularly at users parking their cars in multi-storey car parks. When returning to their car, the user could check the location where the car was parked from the memory of the phone.

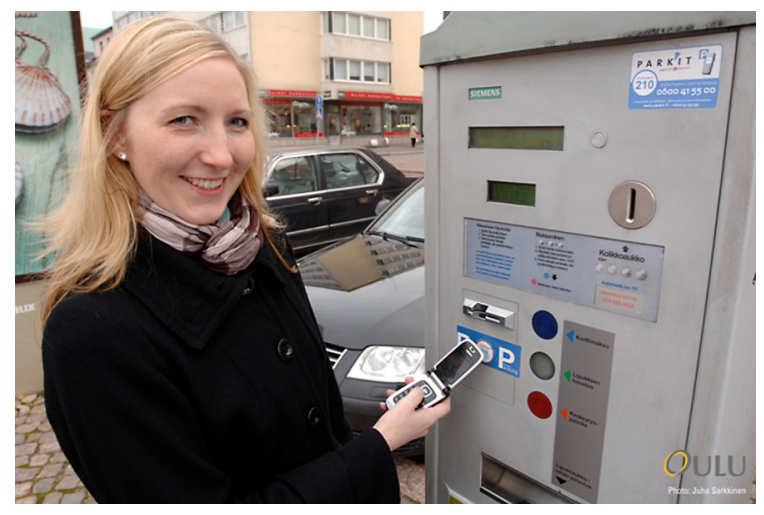

Fig. (1). Parking payment with a mobile. 
The traffic wardens (five users) also had NFC phones, that they could use for checking if a car parked in a paid parking spot had a valid parking payment registered in the background system. They did this by touching the tag located in the car, which sent an inquiry to the background system that triggered a return message indicating if the parking payment was valid.

The trial users were recruited from amongst people who needed to use paid parking at least four times a week. The average age of the trial users who returned the feedback questionnaire (48 out of 51 users) was 47 years. The youngest user was 26 and the oldest 67 years old. $22(43 \%)$ of the users were female and 29 (57\%) male.

\section{Theatre Trial}

In this trial, theatre tickets were stored in mobile phones. When the theatregoers arrived at the venue, the usher validated their tickets by touching the phone of each customer with a mobile phone. The usher's phone acted as a reader, as it validated the ticket stored on the mobile phone of the user. This kind of user interface differs from the UIs described above in two major ways: (1) two similar devices interact with each other, and (2) the devices are used by two users, which adds a greater social dimension to interaction.

The goal of the ticketing application was to better integrate the ticket issuing process with the electronic ticket purchasing process. A ticket received and stored by a mobile phone could be automatically processed through the web site of the theatre, where the users can reserve seats and buy tickets. Also, the trial investigated if the validation of tickets at theatres could be simplified with mobile tickets that can be checked with a touch gesture.

141 theatregoers used the NFC ticket during the trial period. The users were recruited through companies, i.e. the users visited the theatre with their colleagues. 101 users returned the feedback questionnaire. The youngest was 24 and the oldest was 63 years old, and the average age was 44.5 years. Female users (77 users) outnumbered males (24 users). As the trial period was rather long, and each user went to only one show, the researchers did not meet face-toface with most of the users. The theatregoers were introduced to the trial applications with written instructions. Additionally, theatre personnel provided on-site guidance.

\section{Pub Trial}

In the pub trial, the regular customers of a pub could access mobile content describing the beers and other products by touching tags placed on the tables and at the bar (see Fig. 2). Also, information about special offers was provided through a special tag directed at regular customers only, which was located at the bar counter. In addition, the pub used the mobile channel to send SMS-based marketing information to regular customers. In addition to special content available for the trial users, the users were able to record their arrival at the pub by touching a tag near the front door. This enabled the other regular customers to see who were in the pub at a certain time.

The 19 trial users were recruited from the regular customers of the pub by the pub personnel. Of the users who returned the feedback questionnaire (16 users), the youngest trial user was 26 years old and the oldest was 72 , and the average age was 37 years. 14 of the users were male, and only two were female. Pub personnel chose this gender balance because they thought this reflects the gender breakdown of their regular customers.

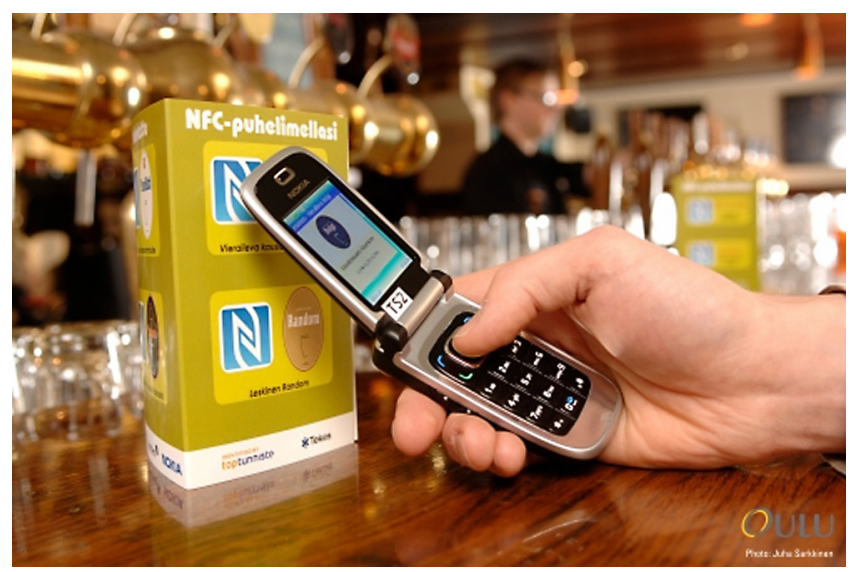

Fig. (2). Accessing mobile Internet content describing the beer selection in a pub.

\section{Restaurant Trial}

In the restaurant trial, the regular lunch customers of a restaurant were given the opportunity to place their meal orders by selecting options from an NFC-enabled menu (see Fig. 3). The goal was to provide faster service to regular customers during busy lunch hours.

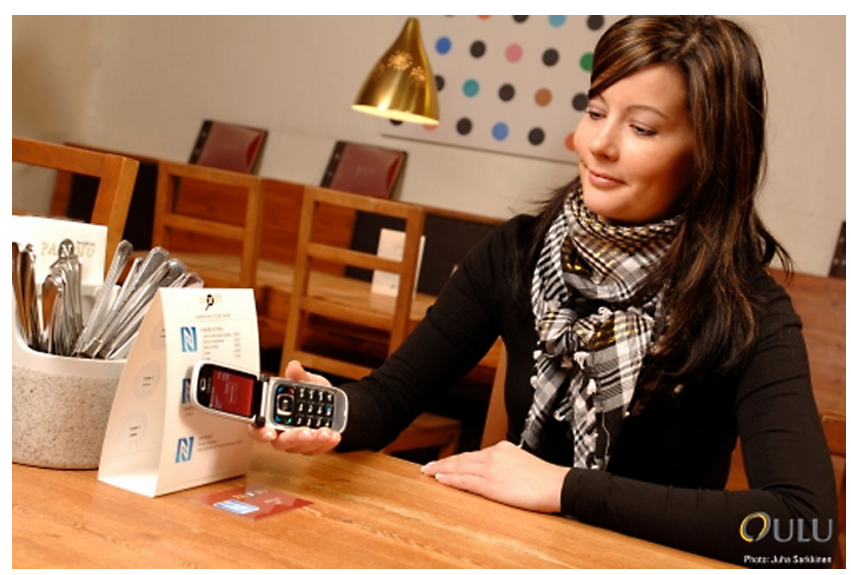

Fig. (3). Ordering lunch by touching a menu.

Each restaurant table was marked with a table identifier tag (see the red tag in Fig. (3) attached to the table in front of the white menu). The user touched this tag to start the ordering process and launch the related application in the phone. An NFC-enabled menu was also placed on each table. The user placed the order by touching the tags for specific menu items. Finished orders were sent to the restaurant cash system and kitchen. The cook could read orders from a screen located in the kitchen and start preparing the meals.

The users could also pay for their lunch by touching the payment machine located at the cashier desk. However, this required the cashier to match the actual order with the payment. The meals were charged through the billing system as usual, i.e. there were no free lunches for the trial users. 
27 trial users were recruited by the restaurant personnel from the regular clients of the restaurant. A total of 23 users returned the feedback questionnaire. The youngest trial user was 22 years old and the oldest 50 . The average age was 37 years. Gender distribution was even.

\section{Information Tags}

In addition to the specific services in the four trials described above, all trial users were able to use information tags distributed in the city centre. Information tags provided trial users with the possibility to access selected mobile Internet content by touching a tag with their mobile phone. A total of 2650 NFC tags were distributed in public places in the heart of one city.

In order to explore the use of information tags in different contexts, the tags were placed in a wide variety of places that were expected to be visited by people with different needs. The information tags were placed:

- $\quad$ on parking meters. The tags placed on parking meters were visible to pedestrians on the pavement. Also, a large amount of people used the parking meters daily, so they would see the tags each time they paid their parking fees.

- $\quad$ inside a theatre. Information tags were placed on the tables where people waited for the play to start or enjoyed refreshments during the intermission. Additionally, information tags were placed on large posters providing information about the plays.

- $\quad$ in a restaurant. Information tags were placed on tables to provide mobile Internet access to the clients.

$\bullet$ in a pub. Information tags were placed on the bar counter and tables for clients.

- $\quad$ in a bus and at bus stops. At the stops, information tags were placed inside the bus shelters. Inside the bus, the tags were placed within reach of seated or standing passengers.

The information tag trial was based on the principle that any user with an NFC-phone could use any of the tags he or she encountered. The tags were not directed at any specific user group, but provided access to generic services that could be useful for anyone. However, for research purposes, trial users were recruited mainly due to the low penetration of NFC-enabled mobile phones. A decision was made to evaluate information tags as an add-on feature with the other NFC-based services described earlier. Therefore, all 238 users who provided feedback about information tags were primarily recruited to evaluate some other service or application concept, and the information tags were provided as an additional service that was available for the trial users. All the users were adults, the youngest being 22 years and the oldest 72 years. The average age was around 40 years.

The tags placed in each location consisted of (1) generic services, such as news, or (2) location-specific services, such as information about the play and the actors at the theatre. The research group designed a combination of both generic and location-specific services for each location. The generic services provided were access to news through the mobile version of the local newspaper, the menu of a local restaurant, the menu of a local pub, the programme of a local theatre, and an operator portal for city-specific information. The selection of services was made primarily from the service offerings of the companies and organizations involved in the research project, not on the basis of a thorough analysis of the needs or requirements of users visiting these specific places. Location-specific content was available at the theatre, bus stops and pub. In the theatre, information about plays - including, for example, trailers and the director's comments - could be accessed through tags placed in posters (see Fig. 4). At bus stops, the users could access real-time bus schedule information through an information tag. In the pub, the clients could use the information tags to access mobile Internet content describing the special selection of beers available.

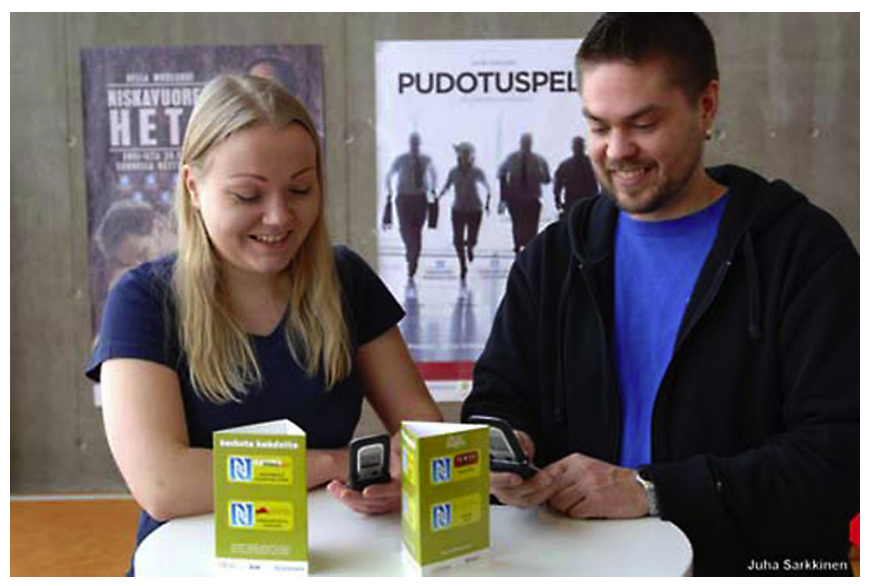

Fig. (4). Information tags in use in the theatre: tag stands on the table and posters with tags in the background.

\section{Data Collection Methods}

Two main sources of data were used for analysis: (1) automatically generated usage logs and (2) questionnaires and related interviews. The logs provided information about who used the tags, which tags were used, and when the tags were used. Subjective experiences were collected with questionnaires and interviews. The design of data collection methods was very similar to the principles described by Consolvo et al. [12] for evaluating ubiquitous computing applications in real-world settings. Pre and post questionnaires and interviews were used in combination with in situ data collection methods, primarily through automated logs, but also with feedback forms and observation.

Subjective user experiences were collected immediately after the trial with questionnaires. In some contexts, a webbased questionnaire was used (e.g. for the theatregoers), and for some, a paper-based questionnaire was applied (e.g. for the parking application users). This choice was made for practical reasons: in the theatre, each user used the NFCenabled mobile phone to access theatre-specific mobile Internet content only during one evening, and they did not meet the researchers face-to-face. Using a web-based questionnaire, the user experience data could be collected immediately after each theatre visit, thus minimizing recall problems. All users returned their online questionnaires within a couple of days after the theatre visit. On the other hand, the parking application users met the researchers in a feedback seminar right after the trial, when they returned their phones to the research team. This provided a perfect 
Table 2. Response Rates for Feedback Questionnaires

\begin{tabular}{|c|c|c|c|c|}
\hline & \# of Returned Questionnaires & Questionnaire Return \% & Questionnaire Type & Time of Returning the Questionnaire \\
\hline \hline Parking & 48 & $94 \%$ & paper combined with web & when users returned the phones \\
\hline Theatre & 101 & $72 \%$ & web & within a couple of days after the visit \\
\hline Restaurant & 23 & $85 \%$ & web & mainly within one week after the trial \\
\hline Pub & 16 & $84 \%$ & web & mainly within one week after the trial \\
\hline
\end{tabular}

opportunity to hand paper questionnaires to the users. They could return these questionnaires when they left the seminar. In the restaurant and pub trials, the request to fill out a feedback questionnaire was sent to the users by email immediately after the trial ended, and a majority of the users replied within one week, and more than half responded on the same day that the request was sent. Table 2 summarizes the feedback questionnaires and response rates.

All questions used in the questionnaires were arranged into three main groups: (1) questions about the gestural interface, e.g. ease of use, locating tags, (2) questions about the trial application, e.g. usefulness and social implications, and (3) questions about information tags, e.g. preferences on content. Each group included both closed questions (for example "ease of use", on a scale from one to five), and open-ended questions meant for providing explanations for the closed questions or for freeform feedback.

Even though the log data and questionnaires provide the primary source of data used in the analysis, we also did some additional, context-specific data collection with a particular view to capturing data that could help in understanding the subjective user experience. The users of the parking application were interviewed after the trial in a feedback seminar. Eleven users volunteered for the interview. The interviews were recorded. The average time of the interview was around ten minutes. Also, the users of the parking application were able to send feedback during the trial using an online feedback form. The total number of feedback forms received during the parking trial was 26 . The researchers observed the theatregoers in actual use situations by attending the theatre with the users. Participatory observation was done during one selected evening, when the researchers went to the theatre and at the same time, trial used the services themselves to get first-hand experience in addition to observing other users and the responses of bystanders. After attending the theatre, the researchers verbalized their own experiences and observations through an open-ended questionnaire. Usability testing was performed in the restaurant pilot. A total of five users used the service in the restaurant, and the testing session was videotaped. In the usability test, the users followed a predefined test script, i.e. they all performed the same activities in the same order. Also, the personnel of each establishment were interviewed about their interpretation of the user experiences evoked by the trial, as they had received feedback from the users while it was in progress and had observed the users on a day-to-day basis.

\section{RESULTS}

In this paper, the material collected in the project was analyzed to construct findings and results on how NFCenabled mobile phones can be used in implementing mobile gestural interfaces for different purposes. The examples of user interfaces constructed and evaluated in the trials illustrate the variety of interface possibilities enabled by integrating NFC into mobile phones.

\section{Physical Browsing}

The NFC tag is able to store a small amount of data that can be read upon touch. NFC standardization [13] specifies how such data stored in the tag needs to be formatted so that the NFC reading device can parse its content and trigger planned behaviour. One of the standardized uses of the data stored in the tag is to trigger Internet access to a specific URL when the NFC device touches the tag.

The interest towards using the tags to access the mobile Internet varied strongly between trials. In the parking trial, only eight users $(17 \%)$ tried tags for Internet access once or more often. On the other hand, at the restaurant, $60 \%$ of users used tags to access Internet content. At the theatre, $58 \%$ of users accessed Internet content using tags. In the pub, all trial users used tags for Internet access, which is not surprising as the services provided in the pub were mostly based on viewing mobile Internet content. As the users had committed themselves to participating in the trial, they naturally enough used the tags. In the other trials, mobile Internet access was more clearly an add-on service.

Fig. (5) represents the distribution of access to generic services available through information tags. It shows clearly that the local news was the most popular and most regularly accessed content. The analysis of the feedback received shows that local news was also rated as the subjectively most interesting content. Local news was received through a local newspaper Internet portal, and it was the content that was updated most frequently. The programme of the local theatre was also rated as interesting in subjective ratings. However, many users were disappointed that the content of the theatre programme was static, as it was not updated during the trial and therefore contained old information. This observation was repeated in several comments; the users expected digital content to be up-to-date, and wished that it would be updated frequently.

Fig. (6) shows how many times the users touched the tags during the trials. The parking payment trial outnumbers the other trials partly because the users touched a tag two or three times each time they parked their cars. Unfortunately, in the beginning, the logs recording activity from generic tags experienced technical problems, impeding their use for the parking pilot. Also, ordering a lunch with the restaurant application required several touches depending on the amount of food items to be ordered. Other user interface solutions were based on one-touch interaction. 


\section{Distribution of content access to generic services}

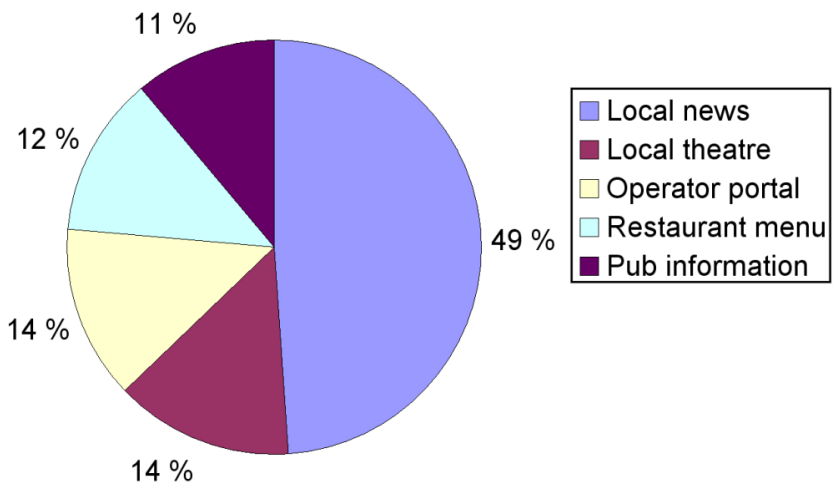

Fig. (5). Distribution of access to Internet content through information tags during a nine-day period estimated not to contain artificial accesses caused, for example, by usability testing or research demonstrations.

Tag usage for generic and trial services

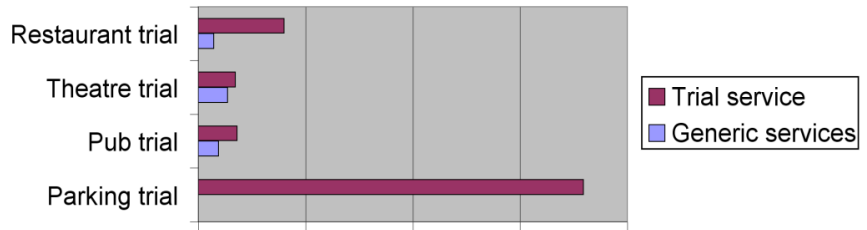

Fig. (6). Number of times the users used tags in each trial. Note that for the parking trial, no reliable data about the usage of generic services is available due to technical problems with logs.

Overall, the users found that learning to use information tags was easy, and they were pleasant to use. $76 \%$ of parking pilot users, $72 \%$ of restaurant pilot users and $67 \%$ of theatre pilot users reported that they learned easily to use tags (selected 5 on a scale of 1 to 5). A majority of the users estimated that they would be interested in using information tags in the future as well, if they would be available for use. Over $50 \%$ of users described their willingness to use tags in the future with "quite interested" or "very interested", except in the restaurant trial, where $35 \%$ selected the option "cannot say" and $42 \%$ selected "quite interested" or "very interested". However, challenges in designing interesting and useful content were identified. As many as $42 \%$ of parking users found the content of information tags "not interesting" ( 1 on a scale of one to five). Theatregoers found the content most interesting, as $58 \%$ of the participants in the theatre pilot found the content "quite interesting" or "very interesting".

\section{Service Access}

The trial services were primarily found to be easy to learn and use. The parking pilot users stated that learning to use the service was very easy (average rating 4.8 on a scale of $1=$ difficult to learn to $5=$ easy to learn; this question was not asked in other trials). Also, a vast majority of the users found the service easy to use. Parking pilot users reported the highest ease of use (average rating 4.8, maximum being $5)$. Even the theatregoers rated the ticket validation service easy to use (average rating 4 on a scale of 1 to 5), even though many experienced problems with ticket validation
(21 out of 102 reported that ticket validation had failed at least once).

In the case of the parking application, the concern that was most frequently mentioned by the trial users was related to the reliability of the system, because the users reported that in some instances they failed to remember to record their departure from the paid parking spot. The application required the user to touch a tag in the car when leaving from paid parking in order to mark the ending of chargeable time. Some users reported that they occasionally forgot to end their parking session. Analysis of logs revealed 67 individual cases (for 23 individual users) that were most probably instances where the user had not ended the chargeable parking session. However, it must be noted that as the users did not actually pay for the service themselves (as the trial services were provided free of charge), they might have been more neglectful in the trial conditions than they would be in a "normal" context when they would need to pay for the service themselves. However, this does not change the fact that users felt that they had problems in remembering to end their parking session, and hoped that the system would aid them in doing this. On the contrary, this worry might be even stronger in conditions where the users would need to pay for their lapse of memory. This is illustrated in the following comments made by parking users:

\section{"It is really easy to remember to start the parking session, but especially towards the end of the trial period I noted that I sometimes did not remember to finish it." \\ "Sometimes I forgot to end my parking session. There should be a reminder or an icon in the phone to indicate that parking payment is running so that incorrect fees can be avoided."}

A similar problem was observed in the pub trial, where the users were able to mark their arrival at and departure from the pub. Many of the users (nine out of 17) commented that they had experienced problems in remembering to record their departure. However, in the pub trial, part of the problem was that some of the users thought that there was no point in marking when they left, and therefore did not see why they should do so. On the other hand, in the opinion of other users, the most valuable feature of the trial service was the possibility to check who is in the pub when considering whether to go there. The attitude towards marking their departure from the pub is well illustrated in the following comment made by a pub trial user:

\section{"Usually I forgot:) On the other hand, this was not a pure accident, as I think I did not get any added value from marking when I left."}

The restaurant trial users estimated that they received faster service with NFC-supported lunch ordering (average rating 1.9 on a scale of $1=$ considerably faster than before to $5=$ considerably slower than before). The users were especially pleased with the food ordering service, but experienced the most problems with the payment feature. As they were able to order the food without the help of the personnel, they seemed to expect that they could complete all the necessary transactions on their own with their phones. However, the trial implementation required the user to pay the cashier. Also, there were technical problems in 
connecting the orders with the payment transaction, which further increased dissatisfaction towards the payment. Payment problems are illustrated in the following comments from the trial users:

\begin{abstract}
"Ordering food was easy and fast. Compared to that, paying felt a bit slow and clumsy. There were sometimes queues at the till ....It could be handy if the payments would be charged directly, for example in a mobile phone bill or with monthly invoicing."

"Why were phone payments processed through the cashier!? Payments should be processed automatically with the order. If we had to wait in a queue to pay, the whole idea of enjoying a quick lunch was wasted."
\end{abstract}

The usability tests done during the restaurant trial revealed the importance of coherent feedback for successful reading activity. Because of design flaws, the feedback varied between tag reading instances - some were confirmed with vibrating feedback, and some were not. The users learned to wait for vibration to confirm successful reading. When they did not receive vibrating feedback, they accidentally selected the same tag several times. This problem became especially evident when selecting menu items to be ordered, as the users accidentally ordered the same food item more than once.

Overall, the users were quite satisfied with the services. On a scale from one to five (five meaning very satisfied), average satisfaction ranged from 3.9 (restaurant trial) to 4.7 (parking trial). However, it must be noted that the parking trial users had free parking during the trial period, which is likely to increase the reported satisfaction. The restaurant trial users were happy to see that the service sped up the ordering process. They also found the user interface easy to use and learn. The most frequent complaint was directed towards payment, which was not as quick, as it required queuing at the cashier and was not well enough integrated with the ordering process. The greatest variety of opinions was seen with respect to the theatre service. Many users stated that the services provided were not interesting or useful.

\section{DISCUSSION}

The findings show that mobile gesture-based user interfaces realized with NFC can provide possibilities to embed user interfaces into the user's everyday environment. Touch facilitates access to content and services by reducing the number of key presses required. However, even though the response time of tag reading is quite acceptable and comfortable, there are still challenges in device-to-device response time and mobile content download. As tag-based user interface infrastructures can be highly distributed and embedded, they are very difficult to control and maintain. The interfaces evolve and change with their surroundings, forming organic user interface infrastructures. Tag placement and the visual design of digital affordances provided through tag-based interfaces can strongly affect the usage patterns and accessibility of tag-based interfaces. These issues are discussed in the following sections.

\section{Fewer Key Presses Needed to Access Content and Services}

Mobile Internet access through touch gestures seems promising, as the user does not need to type or remember the URL needed to access the mobile Internet content. It has been found that the usability of mobile Internet content access increases when the number of key presses required to access and use mobile Internet content is reduced [14, 15]. Reducing key presses is especially relevant in mobile contexts, as the effort required to enter a word on a mobile phone keypad has been measured to be more than double than when typing the same word on a full QWERTY keyboard [15]. With a gestural user interface based on touch, the user does not necessarily need to use the keypad at all and can still be in full control. Also, the user interface paradigm does not require computation or reasoning from the device. These facts separate physical browsing through touch from many other solutions proposed for reducing key presses and facilitating access to mobile Internet services and content, such as context-sensitive search [16] and adaptive content push [17].

Over $90 \%$ of the users stated that it was easy to learn touch-based interaction to access mobile Internet content. It took some time to find the optimal proximity for the gesture - some users preferred to physically touch the tag with their phone, whereas others left some space between the phone and the reader. However, all users were able to learn how to gesture comfortably within a couple of trial touches. A majority of the users also described the use of touching to access Internet content to be "pleasant" $(70-80 \%$ of the users depending on the context).

The parking application implementation was based on a mobile application that communicated with the parking background system via the mobile network. When the users touched parking tags, the application started automatically, and processed the data read from the tags, i.e. the car and location identification, without any direct input from the user. However, the user could also use the keypad of the mobile to enter the location identification. The paid parking areas were identified with location identifiers (such as Center1), and as the trial users were regular users of paid parking, they quickly learned the location identifiers of the parking areas they used. This provided an opportunity to observe whether the users would rather use tags to mark their locations or use the menu and keypad-based user interface to do this. 35 out of 51 users $(66 \%)$ reported that they mostly used the application interface, requiring them to browse menus and use the keypad, whereas 18 users stated that they mostly used tags on parking meters or lamp posts. Log analysis reveals that from the total of 1387 parking instances that took place during the trial, 982 (about 71\%) were reported through the menu and keypad-based interface, and 405 were reported through tags. The users reported that the traditional menu interface was convenient, for example, when it was raining, as it could be used while sitting in the car. They also thought it was fast to use. Location identification through tags was praised for its simplicity and ease of use. Some users said that they used both options for location identification. This is illustrated in the following comment of a parking trial user: 
"Towards the end of the trial I mostly used the menu-based interface, if I was far from the parking meter and heading in another direction."

\section{Response Times}

With a couple of repetitions, all users quickly learned the response time needed to read a tag with a mobile phone, and found the response time to comfortably allow natural gesturing. A vast majority of all trial users described tag usage to be pleasant (around $80 \%$ of all users reported that they found the use of tags to be "quite pleasant" or "very pleasant"). It was observed that it was crucial to provide consistent and coherent feedback to confirm that the tag was read successfully. When there were inconsistencies in tag reading confirmation, the users accidentally made repeated touch gestures (see the description of restaurant usability testing in the "Digital service access" section). The users complained about the long download times needed to view some of the mobile Internet content accessible through touch. This problem was especially pronounced at the theatre, where the location-specific content often included photographs and video, and the monumental concrete structures of the theatre building challenged the mobile network. The following comments from theatregoers illustrate the problems with long content downloading times:

"Reading content through tags worked as I
expected it to work. However, download times
were long, especially for pictures."
"Download times for content were so long that
the phone went to power saving mode before
download was completed."
"Content downloading was too slow. If this
hadn't been a trial, I would not have waited for
the content to download completely."

However, the response times needed for two NFC devices to communicate with each other proved to cause problems for the trial users. Firstly, the actual response time for reading activity between two devices is longer than reading a tag with a mobile phone, and the time needed varies much more. Additionally, the trial settings of the gestural interface based on touching two devices, i.e. the theatre ticketing trial, required two people to interact by touching their mobile phones. The response times were considered too long and unpredictable, which caused uncomfortable social situations. Nervous laughs, restless movements and blushed faces were frequently observed, especially from the theatregoers. Also, a large number of the users commented afterwards that they felt uncomfortable because touching took too long, or that they gave up altogether and decided to show their ticket on the screen of the phone instead of touching. This is illustrated in the following comments from the trial users:

\footnotetext{
"Ticket validation by touching did not work. It was slow and created queues."

"It was difficult to get the ticket validated by the usher."

"The first time we tried to validate the ticket, it did not work."
}

\begin{abstract}
"Ticket validation did not work as planned. However, showing the ticket on the screen of the phone worked fine."
\end{abstract}

As the time needed for successful reading differed from the time needed for reading a tag, the users were not able to predict how long it would take. This increased the discomfort of the theatregoer and raised doubt whether the ticket in the phone was valid in the first place. The ticket checking process seemed to be both slower and less pleasurable with NFC ticketing than traditional paper-based ticketing. Interestingly, the users did not report that they had experienced any feelings of embarrassment or discomfort during ticket validation in their verbal comments in the final feedback questionnaire, even though the observations provided strong indications of these reactions. The user feedback concentrated on reporting about inoperability, slowness and queues.

Of course, all our trial users were first-time users, and with more experience, the situation might change. However, we claim that the first-time experience seen in our trial does not encourage most users to use NFC again. Also, it seems that much of the discomfort was caused by the fact that another person, i.e. the usher, was involved in the time consuming and unpredictable reading process. If the ticket were to be validated by a stand-alone reader, much of the social discomfort would likely disappear.

\section{Organic User Interface Infrastructure}

Constructing a variety of NFC-based user interface infrastructures shows in practice how difficult they are to control. As NFC tags are very cheap, easy to program, easy to distribute, and are all on their own once they are released into the wild, they create an organic user interface infrastructure that evolves with its surroundings. Tag interfaces fixed in static structures evolve in step with their surroundings. Tags in public places are vulnerable to vandalism, they can be fused with other tag-based interfaces, the removal of broken tags can be neglected, etc. As it is practically impossible to control who will create tag interfaces and where, the tag interfaces can become as organic and evolve as uncontrollably as graffiti, i.e. they can form "taggiti" (see Fig. 7).

When tags are broken or unusable, they may even create "tag litter" that is neither valuable nor useful for anyone, and can undermine trust towards tags in general [18]. Even in the relatively short time period of the trials, some users encountered tags that they were not able to read. The users expected that they would get some response from all the tags that were available for touching. Non-functioning tags irritated them. Tags attached to objects that can be easily moved (see the tag interface in the pub trial) can end up in unexpected places and contexts. In the pub trial, the customers noted that the tag stands placed on the tables changed places frequently, and were sometimes difficult to find.

\section{Tag Placement}

One of the factors that seemed to have a strong impact on how reachable and interesting the users found the tags was their placement. In the parking trial, tags were located in places that people visited frequently but very briefly in order 

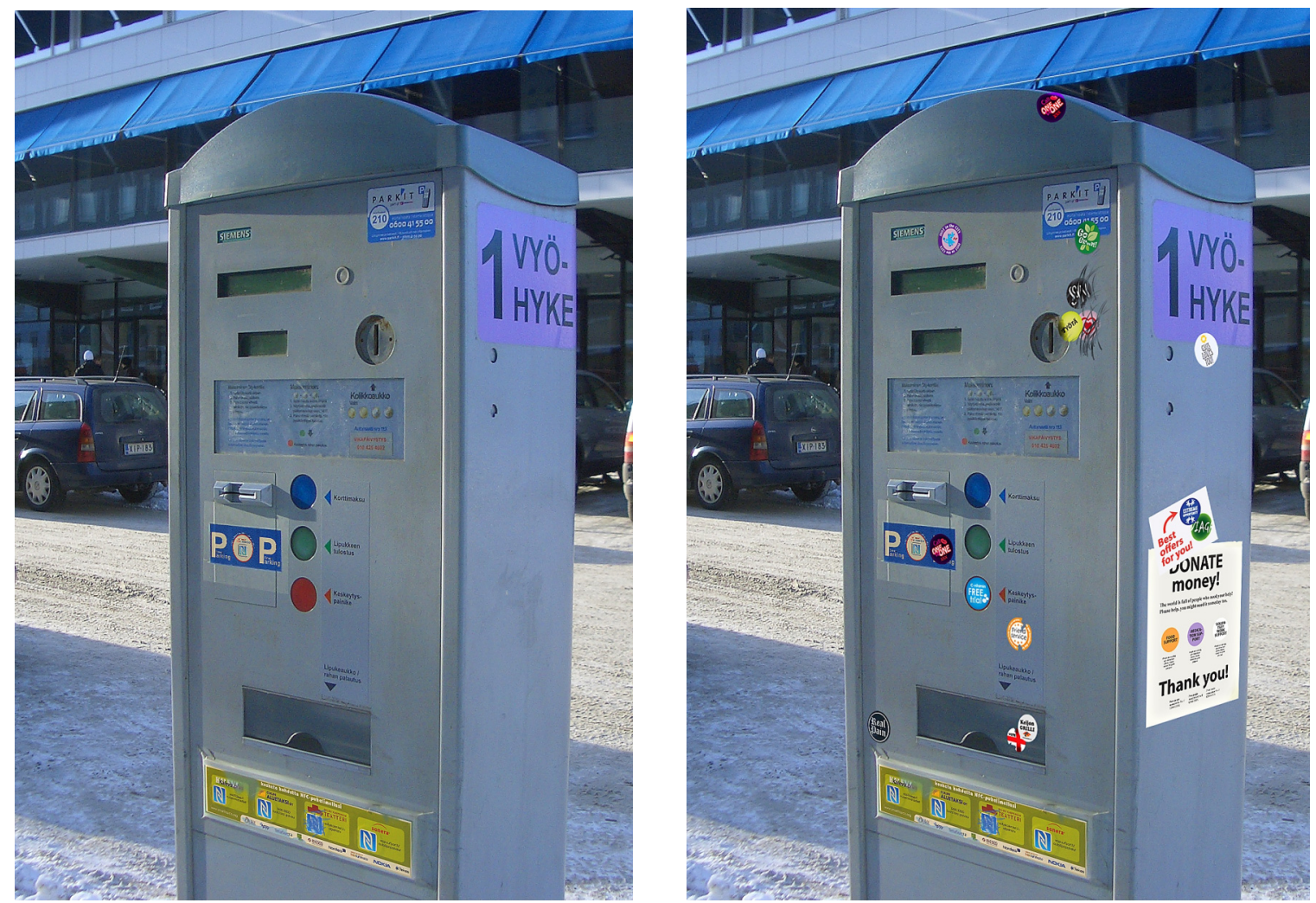

Fig. (7). The picture on the left shows the parking meter with the tag interface designed for the trial. The picture on the right illustrates the same parking meter with "taggiti" (artist's rendering).

to make a short transaction. The users were always on their way somewhere else. These locations were excellent because many people visited them and the tags were within easy reach, providing good opportunities for touch-based interaction. However, as the users only briefly visited the parking meter while on their way to another destination, and had the task of parking payment on their mind, they seemed not to be interested in other kinds of tags - the users would have had to pay attention to notice and read such tags, and then, if they decided to touch the tags, they would have had to devote even more attention and time to downloading and consuming mobile Internet content.

In the restaurant and pub trials, the users were seated at their table and usually were in no hurry. Therefore, they found it considerably easier to notice the tags than did the users of the parking trial, even though the physical distance between the user and the tag and the visual design of the tags were similar. Twenty one percent of the parking trial users reported that they thought that it was either very difficult or difficult to notice information tags, while none of the restaurant and pub trial users selected the options "very difficult" or "difficult" to describe how easy it was to notice information tags.

In the theatre trial, the users also reported that they easily found the tags and their locations $(91 \%$ of the users selected the options "very easy" or "easy"). At the theatre, tags were placed not only on tables (as in the pub and restaurant trials), but also on the foyer walls. Observations indicate that placing tags on the posters on the walls had an effect on how people placed themselves in the space. Normally, people gathered around the centre of the space, where the lighting was brightest and where most of the furniture was located. The space near the walls of the room was more dimly illuminated, and there was no furniture. Some users reported that they had to leave the group they were socializing with in the centre of the room in order to use the tags on the posters on the walls. Also, the users found it more difficult to notice and find the tags on the posters than the tags placed on the tables. In the trial settings, all tags were of about the same size. The standard diameter of a tag is about four centimetres. In locations where tags are placed into objects that are observed from a distance, larger tags could be useful. Of course, the visual indicator of the tag could be large, even if the tag itself is small. However, in this case the users might face problems in locating the tag within the visual indicator.

Choosing the right content to match each location can be a complicated question. If the tag and content providers would like to optimize tag placements, they should observe and analyze how people move and spend time in a space, and what information needs the users might have that can be evoked or inspired by the place or space.

\section{Visual Design}

The tag can be accompanied with visual cues about the content provided. The visual cues can help the user to recognize the digital dimension of the object, to find the right spot to touch, and to identify what content, service or interaction is triggered through touch. Visual cues are particularly important in interfaces that are targeted at casual users and unidentified, large user groups. On the other hand, if the interface is tailored for the regular use of specific 
users, these users probably quickly learn to locate the tag and know what actions touching triggers. Examples could be a tag-based interface targeted at security personnel for marking the places they have visited during their daily round.

In the first trials, a special-purpose visual design was used for each tag. For example, we used an icon representing a bus to mark tags for accessing bus time schedules, and a white $\mathrm{P}$ with a blue background for parking tags (see Fig. 7). However, as the amount of tags and related services grew, the users seemed to need more help in recognizing tags. Therefore, a special visual icon used to mark tag locations was designed. Similar designs have been presented and discussed, for example, by Arnall [19]. Later during the research project, the NFC forum [13] released a visual icon designed to standardize the marking icons, and this was adopted in later trials.

Even though the use of standardized visual language for marking tags seems to help the users to identify and locate tags, there might be cases where it should not be used. If the tag is targeted only at a specific group of users, and requires special software or other resources to operate correctly, users outside the target group might accidentally try to use it if it is marked with a standardized, well-known icon. Encountering tags that do not respond has been found to be frustrating (see the section on "Organic user interface infrastructure" in the discussion).

The visual markings used followed the size of the physical tag, i.e. its diameter was around four centimetres. This seemed to be adequate when the tags were located near the user, i.e. within their reach. However, several users complained that the visual markers were too small when the tags were located further away. This was a particular concern in the theatre pilot, where the posters were placed on the walls of the theatre building. The visual icon was obviously too small to be properly seen from a distance, as is illustrated by the following comments made by the theatregoers:

\section{"Larger target marks should be printed on the posters so that one could see them from a distance." \\ "The tags were too small."}

Of course, the visual marking could be larger than the tag itself. In this case, the users might face difficulties in finding the correct spot to touch. The visual marking should perhaps have two dimensions: a larger icon to indicate that digital properties are available, and a smaller one to mark the actual spot to touch.

\section{Limitations and Validity}

Even though the goal of the field trials was to provide a level of experimental reality [20] that was as high as possible, there are issues in the trial settings that may have and probably have affected the results.

Perhaps the most severe limitation of our research setting was the availability and selection of the services and content accessible through the tags. If NFC readers become common in mobile phones, it is likely that the content and services available through tags will be much more heterogeneous and provided by people and organizations with differing goals and motives.
As the tags were evaluated in a research project, the trial neither set any actual business goals nor sought to serve the public good by providing access to mobile content. Tag placement, design and the accessed information content were not rigorously designed to meet any specific goals, such as optimal coverage of a certain user group. The researchers selected the content provided through tags during a brainstorming session, and the selection criteria used were probably very different from those that would be used if the tags were intended for commercial or any other non-research purposes. As a result, some of the tag content was obviously very badly suited for the specific place it was offered in. For example, many users commented that a tag that helps you call a taxi when you are paying your parking fee was pretty useless. However, badly placed tags were useful for research and design purposes. On the other hand, since the tags are cheap and easy to use by anyone, badly chosen and placed tags will probably be rather common. Some tag providers might find it faster, cheaper and easier to attach tags randomly than to do a proper analysis to determine the optimal tag locations.

Another issue that may have an effect on the results is that due to the low penetration rates of NFC-enabled phones, none of the users were able to use the tags with their own mobile phone. This meant that the users usually carried two mobile phones with them, and used their own mobile as a phone, and the trial phone only for the NFC-enabled features. This might have had an effect on usage frequency, perceived accessibility, user experience and ease of use. Some users reported that they did not want to carry two phones with them, for example, when they went to the pub. Especially in the theatre trial, when the users used the NFC services only on one evening, the users reported difficulties with learning how to operate the user interface of the NFC phone.

The fact that the user experiences were collected in a trial setting probably had an effect on the motivation of the users. The users were recruited as trial users, so they were committed to trying out the services provided. Therefore, the first usage was probably initiated by this commitment, and not purely because of interest or curiosity towards the services provided. In addition, the novelty of the technology and services piqued excitement and curiosity, which probably also has an effect on the results.

Some users received free service during the trial. For example, the users did not pay for the data transfer costs caused by their participation in the trial. This, of course, affects usage and the user experience. The effect was probably strongest in the parking trial, as the users received free parking during the trial period. This was likely to increase satisfaction towards the service in general.

\section{CONCLUSION}

Touching provides a natural user interaction mechanism that, with the emergence of technologies such as RFID (Radio Frequency Identification), has potential to be one of the technologies that can make the visions of ubiquitous computing a reality, as it is predicted to be widely implemented in mobile devices and environments in the near future. This study has explored the adoption and design of mobile touch-based interfaces in everyday interaction. The 
trials and evaluations with more than 300 users brought up issues in adopting and designing touch-based interaction in a mobile environment. It is important to realize that in addition to the device being used, there are also many aspects of the use environment that need to be considered when designing touch-based systems. These include response time, the visual design and placement of tags, and the creation and maintenance of the created environment, e.g. protecting it from becoming useless due to an excess of obsolete tags and "taggitis".

\section{ACKNOWLEDGEMENT}

Research work presented here has received funding from TEKES, the Finnish Funding Agency for Technology and Innovation.

\section{REFERENCES}

[1] Mainwaring S, March W, Maurer B. From meiwaku to tokushita!: lessons for digital money design from Japan: Proceeding of the Twenty-Sixth Annual SIGCHI Conference on Human Factors in Computing Systems, Florence, Italy; April 05 - 10, 2008; pp. 21-4.

[2] Sikka P, Corke P, Valencia P, Crossman C, Swain D, BishopHurley G. Wireless adhoc sensor and actuator networks on the farm: Proceedings of the 5th international Conference on Information Processing in Sensor Networks; Nashville, Tennessee, USA April 19 - 21, 2006; pp. 492-9.

[3] ABI research. Twenty Percent of Mobile Handsets Will Include Near Field Communication by 2012. Retrieved from the website of ABI research; 2007 [2009 May 20] available from: http://www.abiresearch.com/abiprdisplay.jsp?pressid $=838$

[4] Martinussen ES, Arnall T. Designing with RFID: Proceedings of the 3rd international Conference on Tangible and Embedded interaction. Cambridge, United Kingdom, February 16-18, 2009; pp. 343-350.

[5] Ishii H, Ullmer B. Tangible bits: towards seamless interfaces between people, bits and atoms: Proceedings of the SIGCHI Conference on Human Factors in Computing Systems. Atlanta, United States March 22 - 27, 1997.

[6] Klemmer SR, Li J, Lin J, Landay JA. Papier-Mache: toolkit support for tangible input: Proceedings of the SIGCHI Conference on
Human Factors in Computing Systems. Vienna, Austria, April 24 29, 2004; pp. 399-406.

[7] Häikiö J, Isomursu M, Matinmikko T, Wallin A, Ailisto H, Huomo T. Touch-based user interface for elderly users: Proceedings of MobileHCI. Singapore, September 09 - 12, 2007; pp. 289-296.

[8] Ailisto H, Pohjanheimo L, Välkkynen P, Strömmer E, Tuomisto T, Korhonen I. Bridging the physical and virtual worlds by local connectivity-based physical selection. Pers Ubiq Comput 2006; 10: 333-44.

[9] Pradhan S, Brignone C, Jun-Hong C, McReynolds A, Smith MT. Websigns: hyperlinking physical locations to the Web. Computer 2001; 34(8): 42-8.

[10] Konomi S, Roussos G. Ubiquitous computing in the real world: lessons learnt from large scale RFID deployments. Pers Ubiq Comp 2007; 11(7): 507-21

[11] Pering T, Ballagas R, Want R. Spontaneous marriages of mobile devices and interactive spaces. Commun ACM 2005; 48(9): 53-9.

[12] Consolvo S, Harrison B, Smith $\mathrm{I}$, et al. Conducting in situ evaluations for and with ubiquitous computing technologies. Int J Hum Comput Interact 2007; 22(1/2): 103-18.

[13] NFC Forum. Retrieved from the website of the NFC Forum; 2009 [2009 May 20] available from: http://www.nfc-forum.org

[14] Buchanan G, Farrant S, Jones M, Thimbleby H, Marsden G Pazzani M. Improving Mobile Internet Usability: Proceedings of the 10th international Conference on World Wide Web; May 01 05, 2001. Hong Kong, Hong Kong 2000; pp. 673-80.

[15] Kamvar M, Baluja S. (2006). A Large Scale Study of Wireless Search Behavior: Google Mobile Search: Proceedings of the SIGCHI Conference on Human Factors in Computing Systems April 22 - 27, 2006. Montréal, Canada; pp. 701-9.

[16] Church K, Smyth B. (2008). Who, what, where \& when: a new approach to mobile search: Proceedings of the International Conference on Intelligent User Interfaces; January $13-16,2008$. Gran Canaria, Spain; pp. 309-12

[17] Beaver J, Chrysanthis PK, Pruhs K, Liberatore V. To broadcast push or not and what?: Proceedings of the International Conference on Mobile Data Management; May 9-12, 2006. Nara, Japan; pp. 40-5.

[18] Isomursu M. Tags and the City. PsychNol J 2008; 6(2): 131-56.

[19] Arnall T. A graphic language for touch-based interaction: Proceedings of the Workshop on Mobile Interaction with the Real World. September 12, 2006; Espoo, Finland; pp. 18-22.

[20] Aronson E. The Social Animal. 9th Ed. USA: Worth Publishers. 2004. 